\title{
Effects of Brisk Walking Combined with Green Tea Extract on the Aerobic Capacity and Physical Fitness Function in Overweight and Obese Men: A Randomized, Double-Blind, Placebo-Controlled Trial
}

\author{
Tengfei Zhang1, Ningxia Li², Si Chen1, Zhenqing Hou ${ }^{3}$, Atsushi Saito1* \\ ${ }^{1}$ Faculty of Human-Environment Studies, Kyushu University, Fukuoka, Japan \\ ${ }^{2}$ The Center Laboratory, Luohe Central of Hospital, Henan, China \\ ${ }^{3}$ Post-Doctoral Research Center, Luohe Central of Hospital, Henan, China \\ Email: *saito-a@ihs.kyushu-u.ac.jp
}

How to cite this paper: Zhang, T. F., Li, N. X., Chen, S., Hou, Z. Q., \& Saito, A. (2020). Effects of Brisk Walking Combined with Green Tea Extract on the Aerobic Capacity and Physical Fitness Function in Overweight and Obese Men: A Randomized, Double-Blind, Placebo-Controlled Trial. Advances in Physical Education, 10, 207-216. https://doi.org/10.4236/ape.2020.103018

Received: June 12, 2020

Accepted: August 1, 2020

Published: August 4, 2020

Copyright $\odot 2020$ by author(s) and Scientific Research Publishing Inc. This work is licensed under the Creative Commons Attribution International License (CC BY 4.0).

http://creativecommons.org/licenses/by/4.0/

\section{(c) (i) Open Access}

\begin{abstract}
In the present study we investigated the effects of brisk walking combined with GTE ingestion on the physical fitness function and aerobic capacity of inactive overweight and obese men. Participants were randomly assigned to the GTE group (150 mg GTE/tablet, twice/day) or the placebo group. All subjects in the GTE group $(n=12)$ or placebo group $(n=12)$ took part in the 12-week intervention program, and were followed for 4 weeks post-intervention. All variables were measured at week 0 , week 12 and week 16 . We found a significant increase in the aerobic capacity in both groups $(P<0.05)$, and a significant change in the handgrip strength (HS) of the GTE group $(P<0.05)$. After 12 weeks, statistically significant differences were observed in the 8 -foot up-and-go (8FUG); and sit and reach (SR) test results in both groups $(P<$ 0.05 ), while there was no significant change in the one-leg standing with eyes closed results of either group. A down-ward trend was observed after 4 weeks of follow-up. Brisk walking combined with GTE was suggested to have positive effects on aerobic capacity, SR, and 8FUG in overweight and obese men and was more effective in improving HS than brisk walking alone; however, it had no effect on static balance.
\end{abstract}

\section{Keywords}

Brisk Walking, Green Tea Extract, Aerobic Capacity, Physical Fitness, Overweight and Obese Men 


\section{Introduction}

Obesity is not only a major risk factor for chronic diseases, including metabolic syndrome, types 2 diabetes and some cancers, but also reduce the physical fitness functions in daily life (balance, endurance, walking steps) and the lifespan (Hita-Contreras et al., 2013; Lai et al., 2008; Pataky et al., 2014). In addition, obesity is significantly correlated with disability in daily life activities (Forhan, 2009). Increased physical activity is an important component of lifestyle intervention for the management of obesity. Walking, especially brisk walking, has been described as a near perfect "exercise" and has been recommended by health organizations worldwide (Morris \& Hardman, 1997; Nemoto et al., 2007). A systematic review and meta-analysis by Hanson et al. reported that walking had a beneficial effect on body control, VO2max, and aerobic capacity in individuals (Hanson \& Jones, 2015). Garnier et al. mentioned that 16 weeks of brisk walking was more effective than baseline for increasing cardiorespiratory fitness in moderately obese postmenopausal women (Garnier et al., 2015). In addition, Willems Met \& Cook (2018) reported that green tea combined with brisk walking was able to reduce the respiratory exchange ratio and enhanced fat oxidation in women.

Green tea is one of the most popular beverages in the world, especially in Asia. Green tea is rich in catechins, among which epigallocatechin-3-gallate (EGCG) is the most pharmacologically active (Namal Senanayake, 2013). A previous study reported that green tea catechins combined with treadmill running was able to increase the endurance performance in mice (Minegishi et al., 2018). Sae-Tan et al. (Sae-Tan et al., 2014) also showed that GTE combined with voluntary running exercise was more effective for increasing the expression of peroxisome proliferator-activated receptor in skeletal muscle in high-fat fed mice. In addition, Novozhilov et al. (Novozhilov et al., 2015) showed that GTE combined with aerobic exercise was able to improve the aerobic exercise capacity and antioxidant system of erythrocytes. However, the effects of GTE combined with brisk walking on aerobic capacity and physical fitness in overweight and obese men, especially those with under uncontrolled dietary conditions are unknown.

Thus, we hypothesized that GTE combined with brisk walking might increase the aerobic capacity and physical fitness function in overweight and obese men. Therefore, the present study aimed to examine the effects of GTE combined with brisk walking on the change in: 1) aerobic capacity and 2) the balance, strength and other physical fitness function after 12 weeks and 4-weeks follow-up in inactive overweight and obese men.

\section{Methods}

\subsection{Study Design}

This study is a randomized, double-blind, placebo-controlled trial. This study was approved by the Luohe Central Hospital of Clinical Research Ethics Committee (No. 2018-03-015-E01) and has been registered as a clinical trial (Chinese Clinical Trials Registry, ChiCTR1900025134). 


\subsection{Participants}

Twenty-four overweight or obese non-smoking men who were overweight or obese, (age, $39.8 \pm 8.8$ years) volunteered for this study. According to the physical characteristics of Asian, the optimal BMI cut-off value is $24 \mathrm{~kg} / \mathrm{m}^{2}$ for men (Zeng et al., 2014). Those who met the following inclusion criteria were then included: male; between 25 and 59 years of age, BMI between 24 and 32, No participation in any clinical program within the previous 3 months. None were regular consumers of green tea. Individuals with known or suspected cardiovascular disease, diabetes, mental illness, liver, renal disease, and nerve disease were excluded from the study. Participants read and signed an informed consent form that explained the potential risks of this study.

\subsection{Supplement}

GTE and placebo were purchased from Damingtang Company (Hangzhou, China). Each GTE tablet contained $150 \mathrm{mg}$ of EGCG (95\% pure), isomaltitol, and microcrystalline cellulose (MC), and each placebo tablet contained neutral ingredients, such as corn starch, MC and isomaltitol.

\subsection{Intervention}

Twenty-four participants were randomly divided into 2 groups: the GTE combined with brisk walking (GTE) group and the placebo combined with brisk walking (placebo) group. Participants were assigned to either the control or GTE group through stratified randomization according to their body mass index (BMI). All participants performed physical activity irregularly. Enrollment and assignment were carried out by the first author. However, the GTE or placebo assignment was performed by the third assistant. In addition, because studies have demonstrated that GTE is positively correlated with hepatotoxicity, we selected dose of $300 \mathrm{mg}$ EGCG/day, which is considered to be a safe level to ingest (Dekant et al., 2017; Hu et al., 2018). All participants were asked to consume two GTE or placebo tablets daily: one tablet with breakfast and one with dinner. The study assistant supervised the participants who consumed the GTE or placebo by telephone. The GTE tablet contained $150 \mathrm{mg}$ of EGCG, while the placebo tablet contained neutral ingredients such as corn starch, and isomaltitol. The GTE and placebo tablets were, identical in appearance, size and color.

The brisk walking intervention program was conducted for 12 weeks. This outdoor walking program was performed under the supervision of the first author and a research assistant. The walking program involved 12 weeks of brisk walking at an intensity of $65 \%-80 \%$ of the maximum heart rate, which was monitored using a heart rate belt (Murtagh E. M. et al., 2002). Brisk walking was performed 4 times a week, with each session lasting 60 minutes. The warm-up and cool down phases lasted 5 minutes. Due to all subjects being overweight or obese men with physical inactivity, for the first 3 weeks (initiation phase), we set the intensity to $50 \%-65 \%$ of the maximum heart rate for all participants and then gradually increased the intensity. The maximum HR was calculated using 
the equation HRmax $=[220$ - age $]$. All participants were fitted with a heart rate monitor (FS1, Polar, Finland).

\subsection{Physical Fitness Function Testing}

All participants visited the hospital 3 times: at baseline, at 12 weeks after the start, and at 16 weeks after the start (for the 4 -week follow-up). Body weight was measured with a body composition analyser (GAIA KIKO, Korea). Handgrip strength (HS) was measured to the nearest $0.1 \mathrm{~kg}$ using an electronic hand scale (EH101, CAMRY, China). Sit-and-reach (SR), 8-foot up-and-go (8FUG), and eyes closed and one-leg-standing (ECOLS) were measured using relevant professional instruments in a hospital. The 6 minutes walking test (6MWT) was measured indoors in a 30-m hospital corridor. The participants were instructed to walk as far as they could as fast as possible in 6 minutes; encouragement was given every minute during the test period. All parameters were measure at the center laboratory, Luohe Central of Hospital.

\subsection{Statistical Analyses}

All values are presented as the mean and standard deviation. All statistical analyses were performed using the SPSS 20.0 software program (IBM SPSS Statistics, Armonk, USA). An intention-to-treat (ITT) efficacy analysis was performed in this study. We used an analysis of co-variance (ANCOVA), to examine the changes between the groups after the intervention program. After observing the normality, a parametric analysis was performed using a repeated measure analysis of variance ( 2 groups at 3 time-points [baseline, post-test and follow-up]). In all comparisons, $P$ values of $<0.05$ were considered to indicate statistical significance.

\section{Results}

\subsection{Participants and Baseline Characteristics}

An analysis of the baseline characteristics of the participants showed no significant differences between the groups with regard to age, body weight, or BMI. Furthermore, there were no significant differences between the groups in the baseline 6MWT, 8FUG, HS, SR, and ECOLS results (Table 1).

\subsection{The Between-Group ANCOVA}

The changes between groups were analyzed by an ANCOVA, with the preintervention levels as covariates and post-intervention levels as dependent variables. According to the results of the post-treatment ANCOVA, the 6WMT, 8 FUG, and SR results of the two groups did not differ to a statistically significant extent (Table 2).

\subsection{Repeated Assessments}

As shown in Table 3, no significant group, time, or group $\times$ time main effects 
were found in ECOLS. Significant time main effects were found in the 6MWT, 8FUG, HS, and SR results (Table 3). In the placebo group, there were significant changes in the $6 \mathrm{MWT}$ and SR values, and a down-ward trend was observed after 4 weeks of follow-up. In the GTE group, the 6MWT and SR values were significantly reduced, and a downward trend was observed after 4 weeks of follow-up. There was a significant reduction in the 8FUG values after the intervention program; these values increased after follow-up. A similar result was observed in the placebo group. There were no significant groups or group $\times$ time main effects in any of the measurements in the GTE or placebo groups.

Table 1. Subject characteristics at baseline.

\begin{tabular}{ccc}
\hline Variable & GTE group $(\mathrm{n}=12)$ & Placebo group $(\mathrm{n}=12)$ \\
\hline Age (years) & $42.5 \pm 9.82$ & $37.2 \pm 7.2$ \\
Body weight $(\mathrm{kg})$ & $83.0 \pm 9.37$ & $82.3 \pm 8.46$ \\
BMI $\left(\mathrm{kg} / \mathrm{m}^{2}\right)$ & $28.4 \pm 2.24$ & $27.7 \pm 2.26$ \\
6MWT $(\mathrm{m})$ & $615 \pm 62.4$ & $639 \pm 43.0$ \\
8FUG (s) & $4.54 \pm 0.91$ & $4.27 \pm 0.45$ \\
HS (kg) & $45.7 \pm 3.78$ & $45.9 \pm 6.58$ \\
SR (cm) & $1.0 \pm 9.74$ & $6.7 \pm 8.50$ \\
ECOLS (s) & $10.6 \pm 5.55$ & $16.3 \pm 25.24$ \\
\hline
\end{tabular}

No difference between groups was statistically significant. Values are expressed as mean \pm SD. m: meters; s: seconds; kg: kilogram; cm: centimeter; 6MWT: 6 minutes walking test; 8FUG: 8-foot up-and-go; HS: Handgrip strength; SR: Sit-and-reach; ECOLS: eyes closed and one-leg-standing.

Table 2. Changes in the aerobic capacity and physical fitness after 12 weeks' intervention.

\begin{tabular}{|c|c|c|c|c|c|c|c|c|c|c|}
\hline \multirow{3}{*}{ Variable } & \multicolumn{4}{|c|}{ GTE group $(\mathrm{n}=12)$} & \multicolumn{4}{|c|}{ Placebo group $(\mathrm{n}=12)$} & \multirow{3}{*}{$\mathrm{F}$} & \multirow{3}{*}{$P$} \\
\hline & \multicolumn{2}{|c|}{ Unadjusted } & \multicolumn{2}{|c|}{ Adjusted } & \multicolumn{2}{|c|}{ Unadjusted } & \multicolumn{2}{|c|}{ Adjusted } & & \\
\hline & Mean & SD & Mean & SE & Mean & $\mathrm{SD}$ & Mean & SE & & \\
\hline 6MWT (m) & 655 & 70.1 & 666 & 9.3 & 674 & 49.4 & 663 & 9.3 & 0.097 & 0.759 \\
\hline $8 F U G(s)$ & 4.27 & 0.73 & 4.18 & 0.09 & 3.86 & 0.32 & 3.95 & 0.09 & 3.402 & 0.079 \\
\hline $\mathrm{SR}(\mathrm{cm})$ & 4.1 & 9.10 & 7.1 & 1.17 & 10.6 & 6.85 & 7.7 & 1.17 & 0.133 & 0.719 \\
\hline
\end{tabular}

Values are expressed as mean $\pm \mathrm{SD}$.

Table 3. Aerobic capacity and physical fitness analysis of the participants during the experimental and follow-up periods.

\begin{tabular}{cccccccccc}
\hline \multirow{2}{*}{ Variable } & \multicolumn{3}{c}{ GTE group $(\mathrm{n}=12)$} & \multicolumn{3}{c}{ Placebo group $(\mathrm{n}=12)$} & \multicolumn{3}{c}{$P$-value } \\
\cline { 2 - 10 } & Pre & Post & Follow-up & Pre & Post & Follow-up & Group & Time & Group $\times$ Time \\
\hline 6MWT (m) & $615 \pm 15.5$ & $655 \pm 17.5^{*}$ & $640.0 \pm 18.1^{\&}$ & $639 \pm 15.5$ & $674 \pm 17.5^{*}$ & $667 \pm 18.1^{\&}$ & 0.318 & 0.000 & 0.832 \\
8FUG (s) & $4.54 \pm 0.21$ & $4.27 \pm 0.16^{*}$ & $4.31 \pm 0.18^{\#}$ & $4.27 \pm 0.21$ & $3.86 \pm 0.16^{*}$ & $4.02 \pm 0.18^{\sharp}$ & 0.193 & 0.000 & 0.565 \\
HS (kg) & $45.7 \pm 1.55$ & $48.1 \pm 1.35^{*}$ & $46.6 \pm 1.59$ & $45.9 \pm 1.55$ & $47.2 \pm 1.346$ & $47.3 \pm 1.59$ & 0.988 & 0.023 & 0.479 \\
SR (cm) & $1.0 \pm 2.64$ & $4.1 \pm 2.33^{*}$ & $3.4 \pm 2.42^{*}$ & $6.7 \pm 2.64$ & $10.6 \pm 2.33^{*}$ & $9.5 \pm 2.42^{*}$ & 0.085 & 0.000 & 0.777 \\
ECOLS (s) & $10.6 \pm 5.55$ & $17.0 \pm 6.86$ & $19.7 \pm 6.11$ & $16.3 \pm 5.55$ & $20.4 \pm 6.86$ & $20.9 \pm 6.11$ & 0.672 & 0.082 & 0.650 \\
\hline
\end{tabular}

Values are expressed as mean $\pm \mathrm{SE} .{ }^{*},{ }^{*, \&} \mathrm{P} P<0.05 ;{ }^{*} \mathrm{P}$-value for within-group comparisons in pre-and-post period; ${ }^{*} P$-value for within-group comparisons in post and follow-up; ${ }^{\circledR} \mathrm{P}$-value for within-group comparisons in pre and follow-up. 
The results of the measurements of the aerobic capacity and physical fitness function are shown in Table 3. Regarding the physical fitness data, the GTE group exhibited significantly increased 6MWT levels, a significant change in 6MWT also observed in the placebo group. The ECOLS parameters' were not normally distributed (Shapiro-Wilk normality test: $P<0.01$ ). A Wilcoxon test showed no significant differences between the groups or within the groups.

In the GTE group, the 8FUG value decreased from $4.54 \pm 0.21 \mathrm{~s}$ to $4.27 \pm 0.16$ $\mathrm{s}(P<0.01)$ after the 12-weeks intervention program, while HS and SR were increased from $45.7 \pm 1.55 \mathrm{~kg}$ to $48.1 \pm 1.35 \mathrm{~kg}(P<0.01)$ and $1.0 \pm 2.64 \mathrm{~cm}$ to 4.1 $\pm 2.33 \mathrm{~cm}(P<0.01)$, respectively. In the placebo group, the 8FUG value was reduced from $4.27 \pm 0.21 \mathrm{~s}$ to $3.86 \pm 0.16 \mathrm{~s}(P<0.01)$, and there was a significant change in the SR level $(P<0.05)$ after the 12 -week intervention program; these values tended to increase after the 4 -week follow-up period.

\section{Discussion}

In the present study, we found significant changes in the 6MWT values of both groups after the 12-weeks intervention program and a downward trend after 4 weeks of follow-up. Animal and human studies reported that the mechanism underlying the improvement in endurance capacity by GTE combined with aerobic exercise was an increase in fat oxidation and a glycogen-sparing effect (Murase et al., 2005; Sae-Tan et al., 2011). Minegishi et al. (Minegishi et al., 2018) reported that a dietary intake of $0.5 \%$ green tea catechins increased fatty acid oxidation in skeletal muscle and improved the endurance capacity in mice. In a human study, Ichinose et al. (Ichinose et al., 2011) reported a significant reduction in the respiratory exchange ratio during endurance training in healthy male adults after 10 weeks of consuming a supplement containing green tea catechins $(572.8 \mathrm{mg} /$ day), while there was no change in a placebo group. This indicated that green tea catechins combined with aerobic exercise could increase whole body fat utilization during exercise. The findings of this study were contrary to those of a previous study, which showed that the daily intake of $570 \mathrm{mg}$ of tea catechins combined with twice week aerobic exercise significantly altered the aerobic capacity of healthy male adults (Ota et al., 2016). Taken together, these studies suggest that the daily ingestion of high-dose GTE may improve aerobic capacity in overweight and obese men. Therefore, the discrepant results between the present study and the conclusion of the previous studies may be due to the following: first, the present study used a dose of only $300 \mathrm{mg}$ EGCG/day. The second important confounding factor may be the diet. We did not control the diet in the present study.

The present study found a significant increase in HS in the GTE group and a tendency toward an increase in the placebo group. Our study results were in line with the current double-blind controlled trials, which showed that a 10-week exercise program had a beneficial effect on muscle mass in overweight and obese women, even there was no significant difference between groups (Smith et al., 2010). The significant change in the GTE group might have been because the 
ingestion GTE improved muscle recovery after exercise. A study by Panza et al. (Panza et al., 2016) also showed that tea consumption and eccentric exercise was able to hasten the strength recovery for $24 \mathrm{~h}$ after exercise. Another study showed that tea catechins and walking had a significant effect on muscle strength and walking ability in elderly women (Kim et al., 2013). The results of our study might have been due to a positive effect of GTE on muscle strength.

The results of the present study showed significant differences in the 8FUG and SR values of both groups after the 12-week intervention program and a downtrend in the follow-up period. The 8FUG and SR values did not differ between the GTE and placebo groups to a statistically significant extent. These results were in line with those of previous studies, which reported that aerobic exercise was able to improve the physical fitness function in adults (Melam et al., 2016; Ribeiro-Alvares et al., 2018). In addition, in the present study, we did not find a significant change in the ECOLS value after the 12-week intervention program. Although GTE has a positive effect on the body composition, muscle strength, and endurance capacity, no studies have focused on physical fitness in humans. We used these variables to assess the effects on the physical fitness function of the participants in our study. The effects of GTE combined with brisk walking might have been due to an indirect impact on body structure and posture, especially in overweight and obese adults.

The present study was associated with several limitations. First, the sample size was relatively small. Second, we did not set a group of control the dietary patterns of the subjects during the intervention period. Third, an increased number of physiological parameters should be used in a future study. Amulti-arm trial with a larger study population should be conducted to confirm the results of the present study.

\section{Conclusion}

This study showed that the ingestion of GTE combined with brisk walking in inactive overweight and obese men for 12 weeks significantly increased their aerobic capacity and physical fitness, but no significant difference was observed between the groups. We found a significant effect on the HS in the GTE group, which was more effective than brisk walking alone. In comparison to the postintervention values, a downtrend was observed after the follow-up period. Further studies on the effects of exercise intensity, dietary modification and the daily-dose of GTE are needed to clarify the effects of GTE combined with brisk walking on the aerobic capacity and physical fitness function in overweight and obese humans.

\section{Acknowledgements}

This study was supported by Kyushu University Japan and Luohe Central of Hospital China. We are very grateful to all volunteers who took part in this study. 


\section{Funding}

This study was funded by the Kyushu University.

\section{Authors' Contributions}

Tengfei Zhang conceived of this study, designed and coordinated this trial, performed statistical analysis, interpretation and wrote the initial draft of the manuscript; Si Chen performed the randomization and statistical manuscript; Ningxia Li provided medical oversight. Zhenqing Hou performed the data entry. Atsushi Saito provided scientific guidance for the design of the study.

\section{Conflicts of Interest}

The authors declare no conflicts of interest regarding the publication of this paper.

\section{References}

Dekant, W., Fujii, K., Shibata, E., Morita, O., \& Shimotoyodome, A. (2017). Safety Assessment of Green Tea Based Beverages and Dried Green Tea Extracts as Nutritional Supplements. Toxicology Letters, 277, 104-108.

https://doi.org/10.1016/j.toxlet.2017.06.008

Forhan, M. (2009). An Analysis of Disability Models and the Application of the ICF to Obesity. Disability and Rehabilitation, 31, 1382-1388.

https://doi.org/10.1080/09638280802572981

Garnier, S., Vallee, K., Lemoine-Morel, S., Joffroy, S., Drapeau, V., Tremblay, A., Auneau, G. et al. (2015). Food Group Preferences and Energy Balance in Moderately Obese Postmenopausal Women Subjected to Brisk Walking Program. Applied Physiology, Nutrition, and Metabolism, 40, 741-748. https://doi.org/10.1139/apnm-2014-0453

Hanson, S., \& Jones, A. (2015). Is There Evidence That Walking Groups Have Health Benefits? A Systematic Review and Meta-Analysis. British Journal of Sports Medicine, 49, 710-715. https://doi.org/10.1136/bjsports-2014-094157

Hita-Contreras, F., Martinez-Amat, A., Lomas-Vega, R., Alvarez, P., Mendoza, N., Romero-Franco, N., \& Aranega, A. (2013). Relationship of Body Mass Index and Body Fat Distribution with Postural Balance and Risk of Falls in Spanish Postmenopausal Women. Menopause (New York, NY), 20, 202-208. https://doi.org/10.1097/GME.0b013e318261f242

Hu, J., Webster, D., Cao, J., \& Shao, A. (2018). The Safety of Green Tea and Green Tea Extract Consumption in Adults-Results of a Systematic Review. Regulatory Toxicology and Pharmacology, 95, 412-433. https://doi.org/10.1016/j.yrtph.2018.03.019

Ichinose, T., Nomura, S., Someya, Y., Akimoto, S., Tachiyashiki, K., \& Imaizumi, K. (2011). Effect of Endurance Training Supplemented with Green Tea Extract on Substrate Metabolism during Exercise in Humans. Scandinavian Journal of Medicine \& Science in Sports, 21, 598-605. https://doi.org/10.1111/j.1600-0838.2009.01077.x

Kim, H., Suzuki, T., Saito, K., Yoshida, H., Kojima, N., Kim, M., Sudo, M. et al. (2013). Effects of Exercise and Tea Catechins on Muscle Mass, Strength and Walking Ability in Community-Dwelling Elderly Japanese Sarcopenic Women: A Randomized Controlled Trial. Geriatrics \& Gerontology International, 13, 458-465. https://doi.org/10.1111/j.1447-0594.2012.00923.x

Lai, P. P., Leung, A. K., Li, A. N., \& Zhang, M. (2008). Three-Dimensional Gait Analysis 
of Obese Adults. Clinical Biomechanics, 23, S2-S6. https://doi.org/10.1016/j.clinbiomech.2008.02.004

Melam, G. R., Alhusaini, A. A., Buragadda, S., Kaur, T., \& Khan, I. A. (2016). Impact of Brisk Walking and Aerobics in Overweight Women. Journal of Physical Therapy Science, 28, 293-297. https://doi.org/10.1589/jpts.28.293

Minegishi, Y., Otsuka, A., Ota, N., Ishii, K., \& Shimotoyodome, A. (2018). Combined Supplementation of Pre-Exercise Carbohydrate, Alanine, and Proline and Continuous Intake of Green Tea Catechins Effectively Boost Endurance Performance in Mice. Nutrients, 10, 925. https://doi.org/10.3390/nu10070925

Morris, J. N., \& Hardman, A. E. (1997). Walking to Health. Sports Medicine (Auckland, NZ), 23, 306-332. https://doi.org/10.2165/00007256-199723050-00004

Murase, T., Haramizu, S., Shimotoyodome, A., Nagasawa, A., \& Tokimitsu, I. (2005). Green Tea Extract Improves Endurance Capacity and Increases Muscle Lipid Oxidation in Mice. American Journal of Physiology Regulatory, Integrative and Comparative Physiology, 288, R708-R715. https://doi.org/10.1152/ajpregu.00693.2004

Murtagh, E. M., Boreham, C. A., \& Murphy, M. H. (2002). Speed and Exercise Intensity of Recreational Walkers. Preventive Medicine, 35, 397-400.

https://doi.org/10.1006/pmed.2002.1090

Namal Senanayake, S. P. J. (2013). Green Tea Extract: Chemistry, Antioxidant Properties and Food Applications-A Review. Journal of Functional Foods, 5, 1529-1541. https://doi.org/10.1016/j.jff.2013.08.011

Nemoto, K., Gen-no, H., Masuki, S., Okazaki, K., \& Nose, H. (2007). Effects of High-Intensity Interval Walking Training on Physical Fitness and Blood Pressure in Middle-Aged and Older People. Mayo Clinic Proceedings, 82, 803-811. https://doi.org/10.4065/82.7.803

Novozhilov, A. V., Tavrovskaya, T. V., Voitenko, N. G., Maslova, M. N., Goncharov, N. V., \& Morozov, V. I. (2015). Efficacy of Green Tea Extract in Two Exercise Models. Bulletin of Experimental Biology and Medicine, 158, 342-345. https://doi.org/10.1007/s10517-015-2757-4

Ota, N., Soga, S., \& Shimotoyodome, A. (2016). Daily Consumption of Tea Catechins Improves Aerobic Capacity in Healthy Male Adults: A Randomized Double-Blind, Placebo-Controlled, Crossover Trial. Bioscience, Biotechnology, and Biochemistry, 80, 2412-2417. https://doi.org/10.1080/09168451.2016.1224638

Panza, V. P., Diefenthaeler, F., Tamborindeguy, A. C., Camargo Cde, Q., de Moura, B. M., Brunetta, H. S., Sakugawa, R. L. et al. (2016). Effects of Mate Tea Consumption on Muscle Strength and Oxidative Stress Markers after Eccentric Exercise. British Journal of Nutrition, 115, 1370-1378. https://doi.org/10.1017/S000711451600043X

Pataky, Z., Armand, S., Muller-Pinget, S., Golay, A., \& Allet, L. (2014). Effects of Obesity on Functional Capacity. Obesity (Silver Spring, Md), 22, 56-62. https://doi.org/10.1002/oby.20514

Ribeiro-Alvares, J. B., Marques, V. B., Vaz, M. A., \& Baroni, B. M. (2018). Four Weeks of Nordic Hamstring Exercise Reduce Muscle Injury Risk Factors in Young Adults. The Journal of Strength \& Conditioning Research, 32, 1254-1262. https://doi.org/10.1519/JSC.0000000000001975

Sae-tan, S., Grove, K. A., \& Lambert, J. D. (2011). Weight Control and Prevention of Metabolic Syndrome by Green Tea. Pharmacological Research, 64, 146-154.

https://doi.org/10.1016/j.phrs.2010.12.013

Sae-Tan, S., Rogers, C. J., \& Lambert, J. D. (2014). Voluntary Exercise and Green Tea Enhance the Expression of Genes Related to Energy Utilization and Attenuate Metabolic 
Syndrome in High Fat Fed Mice. Molecular Nutrition \& Food Research, 58, 1156-1159. https://doi.org/10.1002/mnfr.201300621

Smith, A. E., Lockwood, C. M., Moon, J. R., Kendall, K. L., Fukuda, D. H., Tobkin, S. E., Cramer, J. T. et al. (2010). Physiological Effects of Caffeine, Epigallocatechin-3-Gallate, and Exercise in Overweight and Obese Women. Applied Physiology, Nutrition, and Metabolism, 35, 607-616. https://doi.org/10.1139/H10-056

Willems Met, Ş. M., \& Cook, M. D. (2018). Matcha Green Tea Drinks Enhance Fat Oxidation during Brisk Walking in Females. International Journal of Sport Nutrition and Exercise Metabolism, 28, 536-541. https://doi.org/10.1123/ijsnem.2017-0237

Zeng, Q., He, Y., Dong, S., Zhao, X., Chen, Z., Song, Z., Chang, G. et al. (2014). Optimal Cut-Off Values of BMI, Waist Circumference and Waist: Height Ratio for Defining Obesity in Chinese Adults. British Journal of Nutrition, 112, 1735-1744.

https://doi.org/10.1017/S0007114514002657 